\title{
Amelogenesis imperfecta in the deciduous dentition: effectiveness of a preventive approach
}

\begin{abstract}
Amelogenesis imperfecta is a rare, genetically determined disorder charaterized by the presence of alterations in dental enamel colour, thickness and/or shape. Teeth can exhibit rapid loss, fracture of enamel and hypersensibility, with negative effects on aesthetics and oral functions. Managing amelogenesis imperfecta in deciduous dentition is challenging and there is still no standard of care established. This report focus on the effectiveness of a preventive approach for amelogenesis imperfecta in the deciduous dentition by means of the outcomes assessment in two young patients.
\end{abstract}

Volume 7 Issue 5 - 2017

\author{
Araxi Balian, Angela Malerba, Laura \\ Strohmenger \\ Department of Pediatric Dentistry, University of Milan, Italy
}

Correspondence: Laura Strohmenger, Pediatric Dentistry Department, Dental Clinic, ASST Santi Paolo e Carlo Hospital, University of Milan, Milan, Italy,

Email laura.strohmenger@unimi.it

Received: July 06, 2017 | Published: July II, 2017

\section{Discussion}

Amelogenesis imperfecta (AI) is a genetically determined disorder characterized by defects in dental enamel development in the absence of other systemic syndromes or metabolic disease. $\mathrm{AI}$ is a rare disease, with a prevalence vary from $1 / 700$ to $1 / 14000$, depending on the population studied. AI is caused by the altera

tion, inherited or sporadic, of the expression of one or more genes encoding for proteins involved in the process of production, mineralization and maturation of dental enamel, such as ameloblastin, enamelin, tuftelin and amelogenin. AI usually affects almost all of the teeth of both the deciduous and the permanent dentition. Clinical sign and symptoms can widely vary: dental enamel can be hypoplastic and/or hypomineralized, so that the teeth presents discoloration, from white to yellow or brown, rough and irregular surface and are more likely prone to wear. In severe cases, teeth exhibit rapid loss and disintegration of part or entire crown under common masticatory forces (post-eruptive breakdown), with reduction of vertical dimension, increase tooth sensibility, difficulty in masticatory function and poor aesthetics. Nevertheless, the increasing plaque retention, due to the difficulty of properly clean the irregular surface of the teeth and the presence of subgingival fracture of dental crown, lead to recurrent marginal gingivitis. Management of AI in paediatric patients is very challenging due to the poor cooperation and the everchanging dentition. So far there is a lack of evidence on this topic and there is still no standard of care established for the treatment of AI in young patients. This paper report the case of two sister affected by different stage of the same type of AI in the decidous dentition and the effectiveness of a preventive approach.

\section{Case description}

Two sister aged 3 and 5 years old were referred to the Paediatric Dentistry Department, Dental Clinic, ASST Santi Paolo e Carlo Hospital, University of Milan for evaluation of dental defects affecting all deciduous teeth. Medical history was non-contributory, since blood exam and ecography of kidneys had excluded the presence of metabolic and/or systemic disease. The father was reported to be affected by AI and this was a probing element for the provisional suspect of AI. Extraoral examination revealed an "edentulous-like" aspect of the face with prognathic appearance, decreased lower facial high and narrowing of the lips. On intraoral inspection, the two patients have full deciduous dentition and presented different stage of a similar pattern of alteration that can be related to an hypoplastic type of AI. The 3-year-old patient showed the presence of milky and brown teeth due to a generalized reduction of enamel thickness, that was almost completely absent in the upper and lower deciduous incisor (Figure 1). Her 5 year old sister showed an advanced stage of AI with severe reduction of dental clinical crown, in some tooth so important that the pulp was even exposed, and the presence of active lesion due to the superinfection of cariogenic bacteria (Figure 2 ). Both the patients presented with marginal gingivitis and poor oral hygiene. When asked, parents said the sisters were used to a frequent intake of sugar through candies and juice. The patients complained increase teeth sensitivity, especially when brushing and eating cold or acid food and drinks. Since the collaboration of both the children was poor, the treatment objectives were planned on a preventive basis and focused on avoiding further loss of tooth structure, eliminating tooth sensitivity, maintaining adequate vertical dimension and perimeter and improving periodontal health. ${ }^{1-4}$

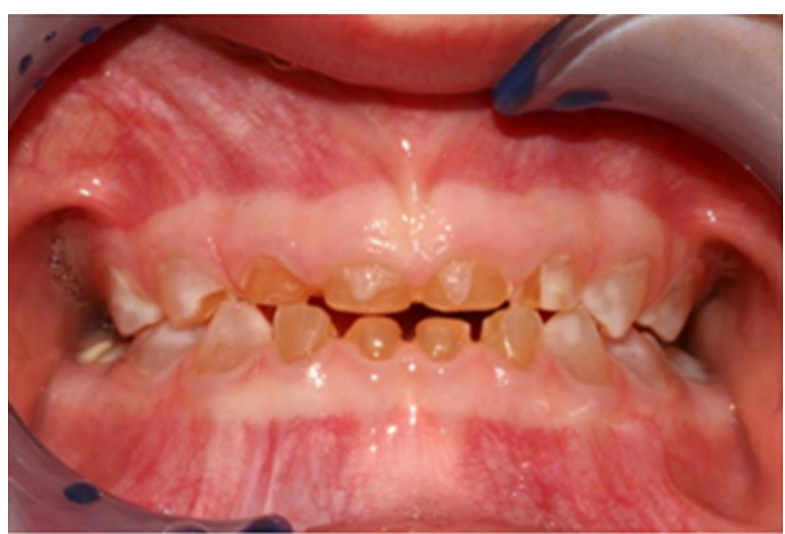

Figure I Early stage of hypoplastic $\mathrm{Al}$ in the 3 years old patient. 


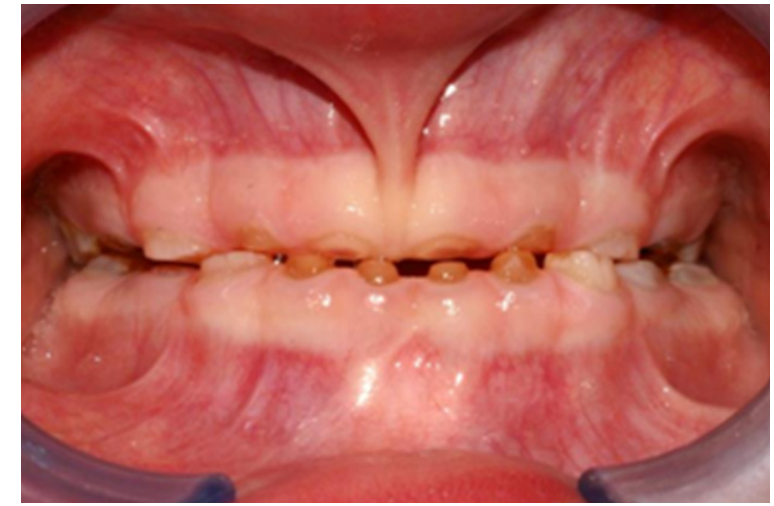

Figure 2 Advance stage of hypoplastic Al in the 5 year old sister.

The preventive approach was based on improvement of oral hygiene and prophylaxis with fluoride. The patients and their parents were instructed to apply an effective technique for tooth-brushing, in order to properly reduce plaque retention without stimulate pain or discomfort. Parents were then motivated to restrict the intake of sugar, highlighting that if they did not cooperate, every treatment effort would have been useless. Fluoride is claimed to have a triple action of reinforcing tooth enamel, promoting remineralization of the teeth and prevent dental caries. The patients undertaken an initial protocol of application of a fluoride varnish every night at home (Elmex fluid ${ }^{\circ}$, GABA, $10.000 \mathrm{ppm})$. After six months of treatment, some active lesion of the 5 year old patient was stabilized, but tooth hypersensitivity remains unchanged in both the patients. A professional application of fluoride was then planned: each sister undertook three consecutive applications of a high concentrated fluoride varnish (Duraphat ${ }^{\circledR}$, Colgate, $22.600 \mathrm{ppm}$ ). After one week the patients reported an improvement in tooth sensitivity and the ability of eat cold and acid food like strawberry, tomatoes and ice-creams. The improvement achieved reveal to be stable as after 3 months the patients reported no relapse of previous symptoms. This report demonstrate that preventive measure and prophylaxis with fluoride are effective in approaching AI in very young patients since they reduce symptoms associated with dental damage and prevent superinfection of cariogenic bacteria, thus avoiding further damage of teeth and reducing negative outcomes on daily life.

\section{Funding}

None.

\section{Acknowledgments}

None.

\section{Conflicts of interest}

The author declares that there is no conflict of interest.

\section{References}

1. Dashash M, Yeung CA, Jamous I, et al. Interventions for the restorative care of amelogenesis imperfecta in children and adolescents. Cochrane Database Syst Rev Jun. 2013;6:1-21.

2. Chen CF, Hu JC, Estrella MR, et al. Assessment of restorative treatment of patients with amelogenesis imperfecta. Pediatr Dent. 2013;35(4):337342.

3. Pousette Lundgren G, Morling Vestlund GI, Trulsson M, et al.A Randomized Controlled Trial of Crown Therapy in Young Individuals with Amelogenesis Imperfecta. J Dent Res. 2015;94(8):1041-1047.

4. McDonald S, Arkutu N, Malik K, et al. Managing the paediatric patient with amelogenesis imperfecta. Br Dent J. 2012;212(9):425-428. 